\title{
Potentially clinically relevant concentrations of Cefazolin, Midazolam, Propofol, and Sufentanil in auto-transfused blood in congenital cardiac surgery
}

Gerda A. Zeilmaker-Roest ${ }^{1,2 *}$, Annewil van Saet ${ }^{3}$, Joost van Rosmalen ${ }^{4}$, Soma Bahmany ${ }^{5}$, Antony van Dijk , Enno D. Wildschut ${ }^{2}$, Dick Tibboel ${ }^{2}$ and Ad J. J. C. Bogers ${ }^{1}$

\begin{abstract}
Background: Use of donor blood in congenital cardiac surgery increases the risk for post-operative morbidity and mortality. To reduce the need for allogenic blood transfusion a technique for peri-operative mechanical red cell salvage is applied. Blood from the operation site is collected in a reservoir, processed, passed through a lipophilic filter and returned to the patient. Influence of this cellsaver system on coagulation, fibrinolysis and inflammatory markers is known. To our knowledge no studies have been performed on the effects of autotransfusion on drug concentrations. A clinically relevant drug dose could potentially be returned to the patient through the autotransfused blood, leading to unwanted drug reactions post-operatively. We aimed to measure drug concentrations in blood salvaged from the operation site and in the auto-transfused blood to determine if a clinically relevant drug dose is returned to the patient.
\end{abstract}

Methods: The study was performed at the Department of Cardiothoracic Surgery of a tertiary university hospital. Blood samples were taken from the reservoir, after processing before the lipophilic filter, the auto-transfused blood, and the waste fluid. Samples were stored at $-80 \mathrm{C}$ and drug concentration for sufentanil, propofol, midazolam and cefazolin were measured using liquid chromatography-tandem mass spectrometry (LC-MS/MS). Drug concentrations measured in the reservoir and the auto-transfused blood were compared and the relative reduction was calculated for each patient.

Results: Blood samples were taken from 18 cellsaver runs in 18 patients, age 0-13 years. Drug concentrations in the reservoir were comparable to concomitant concentrations in the patient. For sufentanil 34\% (median, IQR 2750) of drug concentration was retained from the reservoir in the auto-transfused blood, for midazolam 6\% (median, IQR 4-10), for cefazolin 5\% (median, IQR 2-6) and for propofol 0\% (median, IQR 0-0) respectively.

Conclusion: Depending on the drug, up to $34 \%$ of the drug concentration salvaged from the operation site is returned to the patient through autotransfusion, potentially causing unwanted drug reactions post-operatively. Additionally, influence of a cellsaver system should be considered in pharmacological research during and after congenital cardiac surgery and could result in dose adjustments in the postoperative phase.

Trial registration: Registration at the Dutch Trial Registry (NTR3579) at August 142012.

Keywords: Cardiac surgery, Autotransfusion, Cellsaver, Paediatric, Pharmacology, Intensive care

\footnotetext{
* Correspondence: g.zeilmaker@erasmusmc.nl

'Department of Cardiothoracic Surgery, Erasmus Medical Center, Rotterdam,

The Netherlands

${ }^{2}$ Intensive Care and Department of Pediatric Surgery, Erasmus Medical Center

- Sophia Children's Hospital, Rotterdam, The Netherlands

Full list of author information is available at the end of the article
}

(c) The Author(s). 2018 Open Access This article is distributed under the terms of the Creative Commons Attribution 4.0 International License (http://creativecommons.org/licenses/by/4.0/), which permits unrestricted use, distribution, and reproduction in any medium, provided you give appropriate credit to the original author(s) and the source, provide a link to the Creative Commons license, and indicate if changes were made. The Creative Commons Public Domain Dedication waiver (http://creativecommons.org/publicdomain/zero/1.0/) applies to the data made available in this article, unless otherwise stated. 


\section{Background}

Allogenic donor blood is used in almost all small patients undergoing congenital cardiac surgery. This is mainly due to hemodilution caused by the use of cardiopulmonary bypass $(\mathrm{CPB})$. The technique of mechanical red cell salvage is applied during congenital cardiac surgery. Equipment designed to undertake this task is routinely referred to as an autotransfusion or cellsaver system. Using the cellsaver system blood from the operation site is collected in a reservoir, processed, passed through a lipophilic filter after which it is returned to the patient. The use of donor blood during surgery increases post-operative morbidity, mainly infections [1]. The cellsaver system is used to reduce the need for allogenic blood transfusion and may therefore improve the outcome after surgery. The auto-transfused blood is usually returned to the patient postoperatively on the paediatric intensive care unit (PICU).

Influence of this cellsaver system on coagulation, fibrinolysis and inflammatory markers is known $[2,3]$. In contrast, published literature on the effects of blood loss, volume replacement and use of a cellsaver system on plasma drug concentration is limited. Sue et al. investigated the effect of surgical blood loss and fluid replacement on antibiotic pharmacokinetics in adult patients during cardiac surgery and determined the total cefazolin plasma concentration at several time points [4]. In their study a cellsaver system was used in six of eight patients. Reinfusion of cefazolin-containing blood did not appear to substantially effect post-operative plasma cefazolin concentration in this study. However, cellsaver use was not specified per patient and fluid management during surgery will have changed since 1989. The authors state that major pharmacological effects may occur through infusion of auto-transfused blood in drugs that are highly protein bound or hydrophilic. Rohling et al. showed that plasma concentration of muscle relaxants were stable in autologous blood that was predonated after induction of anaesthesia and returned by the end of surgery without processing with a cellsaver system. Recurarization occurred in two out of 18 studied patients [5].

To our knowledge, drug concentration in auto-transfused blood and influence of a cellsaver system on plasma drug concentrations has not been investigated in infants and children. However, based on published literature, highly protein bound or hydrophilic drugs could persist in the auto-transfused blood. In our clinic, propofol, midazolam, sufentanil and cefazolin are routinely used anaesthetic agents that fit this risk profile. Our hypothesis was that potentially relevant drug doses of propofol, midazolam, sufentanil and cefazolin could be returned to the patient through the auto-transfused blood. This influence could be important, because it may lead to unwanted drug reactions post-operatively.
We aimed to measure drug concentrations in blood salvaged from the operation site and in the blood processed by a cellsaver system during congenital cardiac surgery to determine if a clinically relevant drug dose is returned to the patient.

\section{Methods}

The study was performed at the Department of Cardiothoracic Surgery of a tertiary university hospital. Induction and maintenance of anaesthesia was performed by the attending anaesthesiologist as per local protocol. Either inhalation induction was performed with sevoflurane, or intravenous induction with midazolam or propofol, sufentanil and pancuronium. Maintenance of anaesthesia was performed with continuous infusions of sufentanil, midazolam or propofol. Cefazolin was administered before, during and after the surgical procedure. $\mathrm{CPB}$ technique was dependent on patient weight and operation procedure, and will be described extensively in the CPB-PHARM study publications. No patient underwent deep hypothermia or circulatory arrest during the procedure. We used an Electa cellsaver system (Livanova, München, Germany), with a $55 \mathrm{ml}$ processing bowl. Cellsaver processing was done as per local protocol, according to manufacturer's settings. Blood from the operation site was mixed in the suction tubes with heparinized normal saline to prevent clotting in the tubes. Washing of the cellsaver blood was done with $\mathrm{NaCl} 0.9 \%$. After processing, the cellsaver blood was filtered with a Pall Lipiguard blood filter (Haemonetics S.A., Signy, Switzerland), resulting in the end product, the auto-transfused blood. All residual blood volume from the CPB system was also directly transferred to and processed by the cellsaver system after decannulation. The auto-transfused blood is composed according to the standard of the manufacturer, with $55-60 \%$ erythrocytes in $\mathrm{NaCl} 0.9 \%$.

Blood samples were taken from the reservoir, after processing of salvaged blood before the lipophilic filter, the auto-transfused blood, and from the waste fluid. Samples were collected at the end of surgery. If there was a need to run the cellsaver system both during and after surgery, samples were taken after the first run for best comparison. Samples were stored at $4^{\circ}$ Celsius until processing. Samples were centrifuged $(10 \mathrm{~min}$ at $3600 \mathrm{rpm}$ ) and the plasma transferred to polypropylene cryogenic vials with polypropylene screw caps (Sarstedt Aktiengesellschaft \& Co, Nümbrecht, Germany). Samples were stored at $-80^{\circ}$ Celsius until analysis.

Drug concentration were measured at the pharmacological laboratories of the Erasmus MC. A certified research technician from the ISO certified pharmacy laboratory performed the FDA validated drug analyses. In all analyses quality control samples are included, as is obliged in FDA analyses and ISO and GCP certified 
laboratory. Drug concentrations for propofol (Fresenius Kabi Nederland BV, Zeist, the Netherlands), midazolam and midazolam metabolites (Actavis Group PTC ehf., Hafnarfjördur, Iceland) were measured using LC-MS/MS (Waters Corp., Milford, MA, USA). Drug concentration for sufentanil (Hameln Pharma Plus GmbH, Hameln, Germany) and cefazolin (Kefzol ${ }^{\circ}$, Eurocept BV, Ankeveen, the Netherlands) were measured using LC-MS/MS (Thermo Fisher Scientific, Waltham, MA, USA).

Lower and upper limits of quantification (LLOQ and ULOQ, respectively) were: sufentanil, LLOQ $0.25 \mathrm{mcg} /$ L, ULOQ $50 \mathrm{mcg} / \mathrm{L}$; propofol LLOQ $100 \mathrm{mcg} / \mathrm{L}$, ULOQ $25000 \mathrm{mcg} / \mathrm{L}$; midazolam LLOQ $2 \mathrm{mcg} / \mathrm{L}$, ULOQ 2400 $\mathrm{mcg} / \mathrm{L} ; \mathrm{OH}$-midazolam LLOQ $3 \mathrm{mcg} / \mathrm{L}$, ULOQ 2300 $\mathrm{mcg} / \mathrm{L}$, midazolam glucuronide LLOQ $10 \mathrm{mcg} / \mathrm{L}$, ULOQ $3000 \mathrm{mcg} / \mathrm{L}$; cefazolin LLOQ $1 \mathrm{mg} / \mathrm{L}$, ULOQ $100 \mathrm{mg} / \mathrm{L}$. Drug concentrations for propofol were not measurable after auto-transfusion and were treated as zero in the analysis.

\section{Samples size calculation}

The sample size was set at 18 patients. Due to a lack of published literature on this subject, a formal sample size calculation or power analysis was not considered feasible. The chosen sample size of 18 patients should be sufficient to estimate the median and the variability of the drug concentrations with reasonable precision.

\section{Statistical analysis}

The ratio between drug concentrations measured in the reservoir and drug concentrations measured in the auto-transfused blood was calculated for each patient. The distributions of the drug concentrations in the reservoir and in the auto-transfused blood and their ratios were summarized with the median and the interquartile range (IQR) per drug, to give an indication of the percentage of the drug concentration remains in the auto-transfused blood. The correlation between the absolute drug concentrations measured in the reservoir and concentrations in the auto-transfused blood were calculated using Spearman's rank correlation coefficients. Drug concentrations did not follow a normal distribution and were $\log (10)$ transformed in order to calculate the relative reduction. Relative reduction per drug was calculated by subtracting the $\log (10)$ transformed concentration in the auto-transfused blood from the $\log (10)$ transformed concentration in the reservoir. The relative reduction per drug is plotted against the reservoir concentration to predict the relative reduction per starting concentration in Fig. 3a-d, together with a linear regression line. The $\mathrm{R}^{2}$ was used to show the predictive value of the drug concentration in the auto-transfused blood based on the starting concentration in the reservoir, with a $R^{2}$ of 0 representing no correlation and a $R^{2}$ of 1 representing a perfect correlation. The reported $\mathrm{R}^{2}$ and the $95 \%$ confidence interval (CI) in Fig. 3a-d are based on the linear regression of the $\log (10)$ transformed relative reductions on the $\log (10)$ transformed concentrations in the reservoir.

This study was a part of the CPB-PHARM study, investigating pharmacokinetics (PK) and pharmacodynamics (PD) of routinely used drugs in neonates and children during cardiac surgery with the use of cardiopulmonary bypass (approved by IRB Erasmus MC, protocol number 2011-400, Dutch Trial Registry NTR3579). Informed consent was obtained for all study participants according to Dutch law.

\section{Results}

Blood samples were taken from 18 cellsaver runs in 18 paediatric patients. CPB was used in all surgical procedures. Patient characteristics are shown in Table 1. As shown in Table 1, the cellsaver volume processed postoperatively is larger than the total blood loss during surgery because of addition of the residual volume of the $\mathrm{CPB}$. The amount of washing fluid used is mainly dependent on the processed cellsaver volume. Not all patients received all tested drugs (see Table 2).

Patient drug concentrations for sufentanil, cefazolin, midazolam and propofol were measured for the CPB-PHARM study (MEC2011-400). This study investigates the influence of the cardiopulmonary bypass on the PK and PD of routinely used drugs during and after congenital cardiac surgery. Patient drug samples taken from the arterial catheter during surgery were compared to drug concentrations measured in the reservoir. Drug concentrations measured in the reservoir of the cellsaver system before processing were comparable to concomitant concentrations in the patient, as was expected, and are thus clinically relevant doses. Drug levels for the different drugs and compartments are shown in Table 2. For all drugs, the decrease in drug concentration was largest after washing of the cellsaver blood. The effect of the lipophilic filter further decreased drug concentrations in all drugs except sufentanil. Considerable concentrations of all drugs were measured in the waste fluid.

Median sufentanil concentration in the reservoir was $0.27 \mathrm{mcg} / \mathrm{L}$ (IQR 0.18-0.35). Median sufentanil concentration in the auto-transfused blood was $0.10 \mathrm{mcg} / \mathrm{L}$ (IQR 0.09-0.10). Therefore 34\% (median, IQR 27-50) of drug concentration was retained from the reservoir in the auto-transfused blood. Detailed concentrations per sample site and percentage recovery are shown in Table 2 and Fig. 1.

Median midazolam concentrations in the reservoir and the auto-transfused blood were $192.69 \mathrm{mcg} / \mathrm{L}$ (IQR 49.06316.42) and $11.87 \mathrm{mcg} / \mathrm{L}$ (IQR 7.16-20.16) (6\%, median 
Table 1 Patient characteristics

\begin{tabular}{ll}
\hline Patient Characteristic & $\mathrm{N}$ (mean, minimum - maximum) \\
\hline Age & 2 y 6 months (2 months - 13 y 11 months) \\
Female /male & $10 / 8$ \\
Surgical procedure & 6 \\
$\quad$ Correction ASD type 2 & 3 \\
TCPC & 3 \\
Correction TOF & 2 \\
Correction CAVSD & 4 \\
$\quad$ Miscellaneous & $12.6(1.6-62.7)$ \\
Total peroperative blood loss (ml/kg) & $290(135-910)$ \\
Cellsaver volume processed (ml) & $12(3.9-26)$ \\
Cellsaver product after processing (ml/kg) & $783(300-1200)$ \\
Washing fluid used $(\mathrm{ml})$ & \\
\hline
\end{tabular}

Kg: kilogram, ml: millilitre, y: years. Surgical abbreviations: ASD: Atrial Septal Defect, TOF: Tetralogy of Fallot, TCPC: Total Cavopulmonary Connection, CAVSD: Complete Atrioventricular Septal Defect. Miscellaneous: correction Partial Abnormal Pulmonary Venous Return (1), Chauvaud procedure (1), Mitral Valve Replacement (1), Correction Subaortic Stenosis (1)

IQR 4-10) respectively. Details of midazolam recovery are shown in Table 2. Biologically active midazolam metabolites, 1-hydroxy-midazolam and midazolam glucuronide, were also measured. Recovery of 1-hydroxy-midazolam and midazolam glucuronide was $11 \%$ (median, IQR 7-29) and 6\% (median, IQR 4-8) respectively. Details of 1-hydroxymidazolam and midazolam glucuronide recovery are shown in Table 2 and Fig. 1.

Median cefazolin concentration in the reservoir and the auto-transfused blood was 228.44 mg/L (IQR 118.47295.85) and $8.7 \mathrm{mg} / \mathrm{L}$ (IQR 5.13-11.44) (5\%, median, IQR 2-6) respectively. Detailed concentrations per sample site and percentage recovery are shown in Table 2 and Fig. 1.

Median propofol concentration in the reservoir and in the waste fluid was $0.65 \mathrm{mg} / \mathrm{L}$ (IQR $0.21-1.86$ ) and $0.18 \mathrm{mg} / \mathrm{L}$ (IQR 0.11-0.25) respectively. Propofol concentration could not be measured after processing and in the auto-transfused blood. Detailed concentrations per sample site and percentage recovery are shown in Table 2 and Fig. 1.

The correlations between drug concentrations measured in the reservoir and concentrations in the auto-transfused blood are shown in Fig. 2a-e. The relative reduction per drug is plotted against the reservoir concentration to predict the relative reduction per starting concentration in Fig. 3a-e.

\section{Discussion}

To our knowledge this is the first study to measure drug concentrations in auto-transfused blood after paediatric cardiac surgery. Drug recovery varies between drugs in the auto-transfused blood and not all drugs are found in

Table 2 Drug concentration per sample site

\begin{tabular}{|c|c|c|c|c|c|c|}
\hline \multirow[t]{2}{*}{ Drug } & \multirow{2}{*}{$\begin{array}{l}\text { Patients } \\
\text { (n) }\end{array}$} & \multicolumn{4}{|c|}{ Concentration (median, IQR) } & \multirow{2}{*}{$\begin{array}{l}\text { Ratio reservoir } \\
\text { vs } \\
\text { autotransfused } \\
\text { blood in \% } \\
\text { (median, IQR) }\end{array}$} \\
\hline & & Reservoir & $\begin{array}{l}\text { After processing, before } \\
\text { filter }\end{array}$ & $\begin{array}{l}\text { Auto-transfused } \\
\text { blood }\end{array}$ & Waste fluid & \\
\hline Sufentanil (mcg/L) & 18 & $0.27(0.18-0.35)$ & $0.10(0.09-0.12)$ & $0.10(0.09-0.10)$ & $0.13(0.10-0.16)$ & $34(27-50)$ \\
\hline Midazolam (mcg/L) & 18 & $\begin{array}{l}192.69(49.06- \\
316.42)\end{array}$ & $18.68(4.91-29.88)$ & 11.87(7.16-20.16) & $56.48(17.25-75.18)$ & $6(4-10)$ \\
\hline $\begin{array}{l}\text { 1-hydroxy-midazolam (mcg/ } \\
\text { L) }\end{array}$ & 18 & $36.56(9.45-62.74)$ & $6.23(4.39-11.52)$ & $4.67(3.82-5.95)$ & $3.51(2.72-3.84)$ & $11(7-29)$ \\
\hline $\begin{array}{l}\text { Midazolam glucuronide } \\
(\mathrm{mcg} / \mathrm{L})\end{array}$ & 18 & $\begin{array}{l}348.21(236.63- \\
592.21)\end{array}$ & $36.41(15.83-57.13)$ & $29.22(21.40-41.82)$ & $\begin{array}{l}114.07(75.33- \\
189.71)\end{array}$ & $6(4-8)$ \\
\hline Cefazolin (mg/L) & 18 & $\begin{array}{l}228.44(118.47- \\
295.85)\end{array}$ & $10.50(6.63-17.47)$ & $8.70(5.13-11.44)$ & $59.34(40.68-77.80)$ & $5(2-6)$ \\
\hline Propofol (mg/L) & 6 & $0.65(0.21-1.86)$ & $0(0-0)^{*}$ & $0(0-0)^{*}$ & $0.18(0.25-0.11)$ & $0(0-0)$ \\
\hline
\end{tabular}

IQR Interquartile range, $L$ Litre, Mcg Microgram, $m g$ Milligram, n.a. Not applicable *Drug concentrations for propofol were not measurable after autotransfusion and were treated as zero in the analysis 


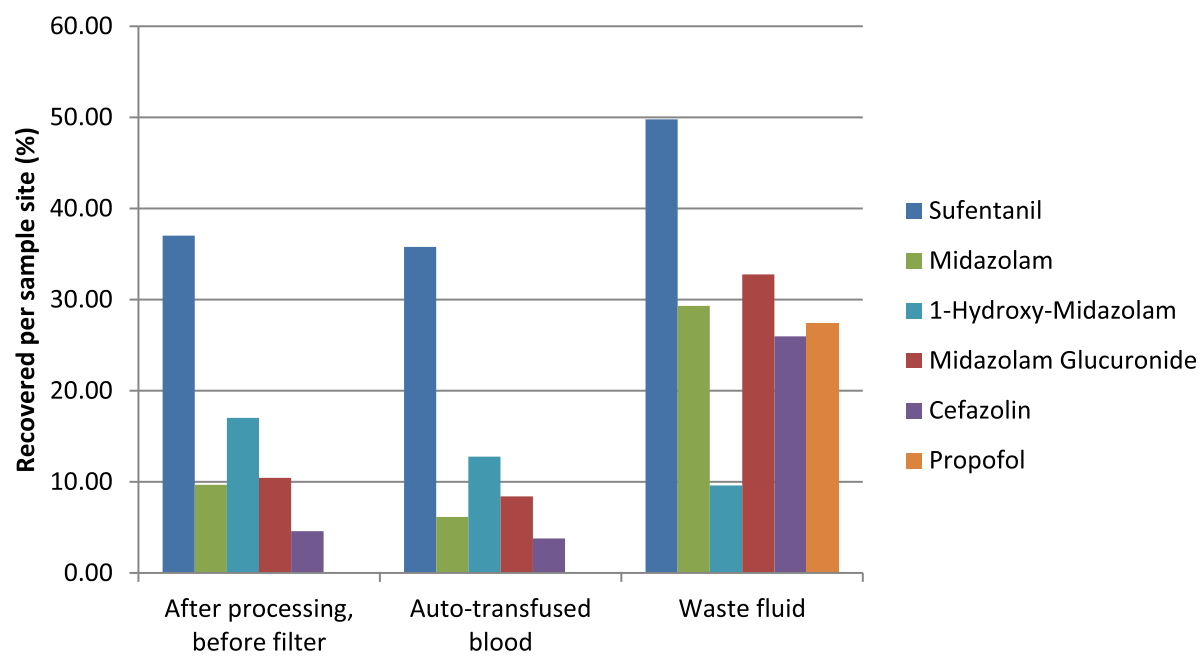

Fig. 1 drug recovery per sample site (\%). Drug concentration in the reservoir is set as 100\%

clinically relevant concentrations. For sufentanil 34\% (IQR 27-50) of drug concentration was retained from the reservoir in the auto-transfused blood, for midazolam 6\% (IQR 4-10), for cefazolin 5\% (IQR 2-6) and for propofol 0\% (IQR 0-0), respectively. For midazolam mainly the 1-hydroxy-midazolam metabolite was recovered in the auto-transfused blood. 1-hydroxy-midazolam is at least as active as midazolam and may contribute to the overall activity of midazolam [6]. The potential influence of the return of auto-transfused blood processed in a cellsaver system on plasma drug concentration in infants and children has not previously been investigated.

Based on the literature by Sue et al. [4] and our own experience with extra corporeal membrane oxygenation (ECMO) circuit characteristics [7], the expectation was that lipophilic drugs would be sequestered in the synthetic components of the cellsaver system and the lipophilic filter and thus be prevented from returning in the auto-transfused blood whereas more hydrophilic drugs could be retained in the auto-transfused blood.

Any lipophilic compounds that are left in the auto-transfused blood after washing should be removed by the lipophilic filter. According to the manufacturer proteins and lipids should be completely washed from the end product so that it contains only erythrocytes and $\mathrm{NaCl} 0.9 \%$. We have shown that washing of the cellsaver blood is the most effective step in clearing drugs from the auto-transfused blood. However both protein bound as well as lipophilic drugs were recovered in the end product. Interestingly sufentanil concentrations were markedly higher compared to the other lipophilic drugs. Drug characteristics of sufentanil, propofol and midazolam regarding protein binding and lipophilicity are fairly similar. With a $\log \mathrm{P}$ of 3.95 , sufentanil is highly lipophilic, with $93 \%$ protein binding, mainly to albumin.
Propofol and midazolam are also both highly lipophilic with $\log$ P's of 3.79 and 3.89 respectively, with a slightly higher protein binding than sufentanil, of 95-99 and $97 \%$ respectively. Therefore lipophilicity or protein binding do not seem to predict drug concentrations in the auto-transfused blood.

Redistribution of lipophilic drugs from erythrocytes into the auto-transfused blood could explain why lipophilic drugs are recovered. Redistribution may occur because of a shift of drugs from the erythrocyte to the $\mathrm{NaCl} 0.9 \%$ solution, or because of haemolysis of the erythrocyte. Also, measuring propofol by the precipitation method instead of LC-MS/MS may have resulted in measurable propofol concentrations after processing. Overall, the recovered absolute drug concentrations of the tested drugs where low. Therefore the absolute differences in plasma drug concentration in the patient and drug concentration in the auto-transfused blood may not be substantial.

The hydrophilic drug, cefazolin, was almost entirety washed from the auto-transfused blood. With a $\log \mathrm{P}$ of -0.58 cefazolin was the most water-soluble drug we have measured. Our results are probably explained by the washing of the cellsaver blood with $\mathrm{NaCl} 0.9 \%$. It is likely that cefazolin dissolved in the $\mathrm{NaCl} 0.9 \%$ solution and was washed from the auto-transfused blood, even though cefazolin concentrations in the waste fluid were low.

Unwanted drug reactions due to auto-transfused blood may not be clinically relevant in all patients.

Most at risk for clinical effects are small patients who have had major cardiac surgery with the use of $\mathrm{CPB}$, where the volume of returned cellsaver blood is relatively large compared to the patient's own circulating volume. Also, due to long $\mathrm{CPB}$ time, organ perfusion 


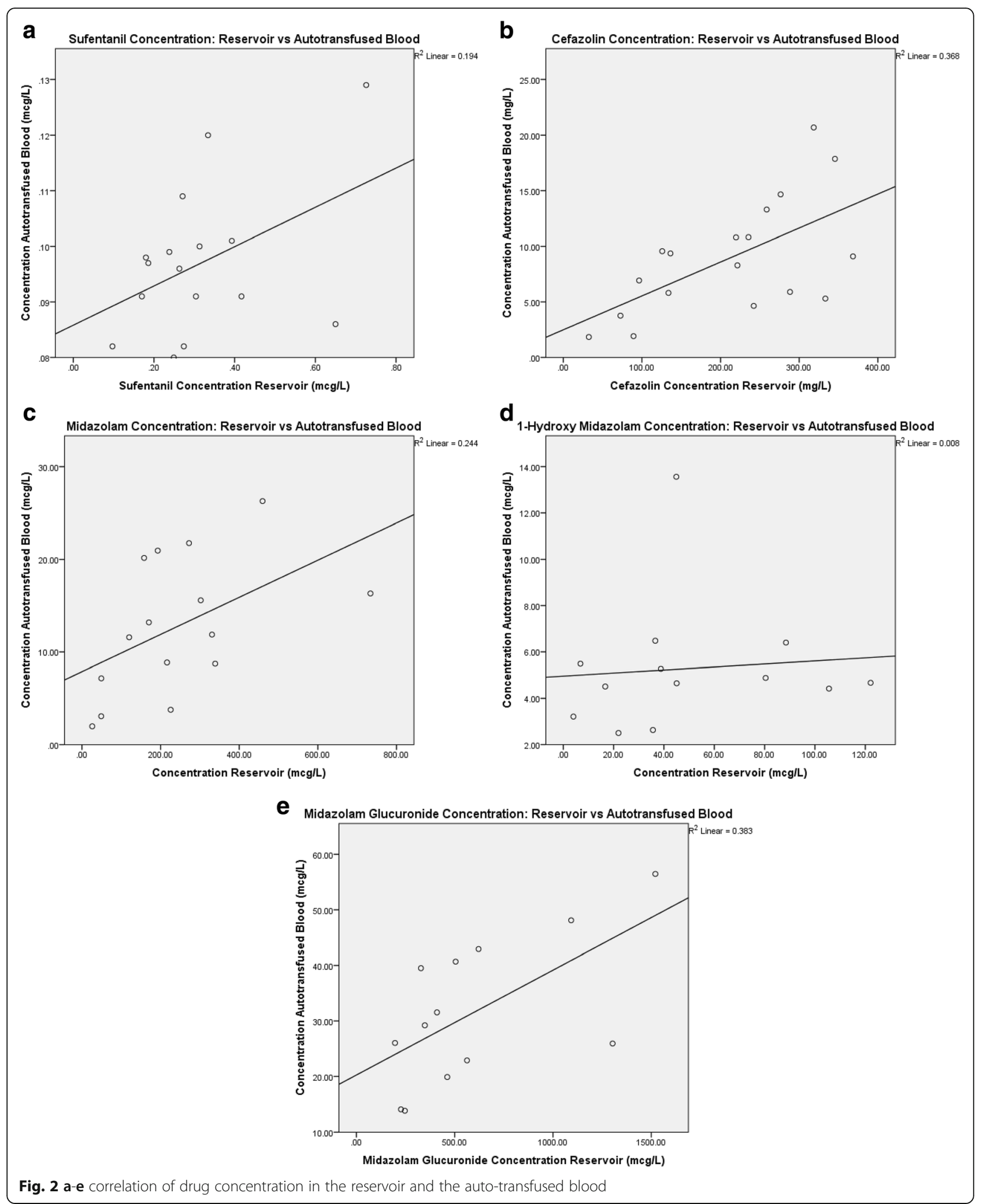



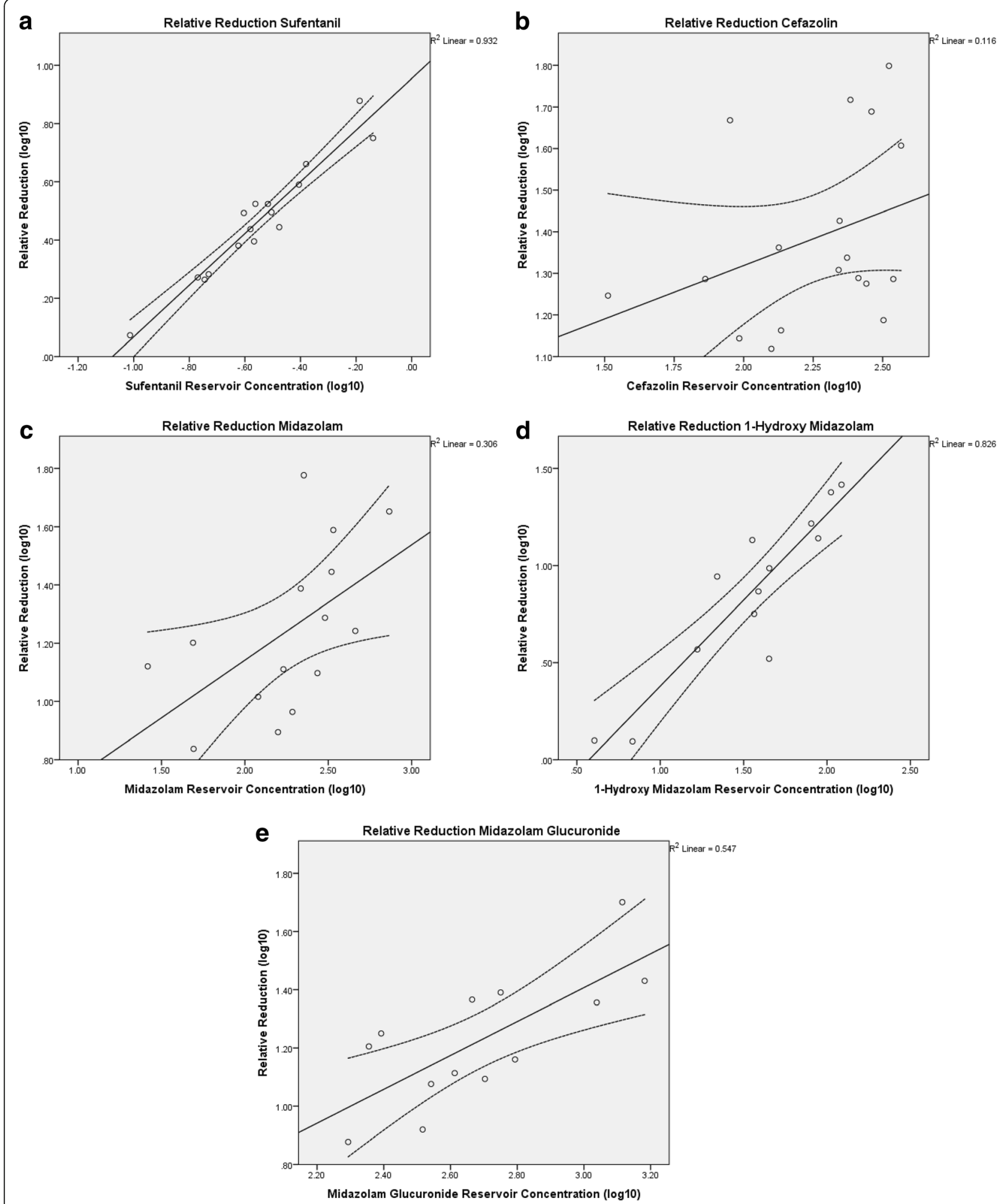

Fig. 3 a-e relative reduction predicted by concentration in reservoir (with dotted line 95\% Cl)

will be decreased, resulting in a lower clearance of drugs from the body [8]. Returning a large volume of auto-transfused blood to a small patient with a decreased drug clearance could lead to an accumulation of drugs, resulting in adverse effects and toxicity especially for sufentanil. Also, the cellsaver blood is generally 
administered as a bolus, rather than a continuous infusion. This may be particularly problematic in patients who are not on mechanical ventilation when the auto-transfused blood is administered.

However, the concentration of sufentanil in the auto-transfused blood is $0.1 \mathrm{mcg} / \mathrm{L}$. In a worst case scenario, when $1 \mathrm{l}$ of auto-transfused blood is returned to a patient with a bodyweight of $3 \mathrm{~kg}$, the sufentanil dose administered with the auto-transfused blood would be $0.033 \mathrm{mcg} / \mathrm{kg}$. The target sufentanil dose at induction of anaesthesia is $0.6 \mathrm{mcg} / \mathrm{kg}$ [9]. Therefore, if the auto-transfused blood is returned to the patient during or very shortly after surgery, the sufentanil concentration in the patient is higher than the sufentanil concentration in the auto-transfused blood and dilution of plasma sufentanil concentration will occur. If the auto-transfused blood is given in a bolus sometime after sufentanil is stopped, a peak in plasma concentration may still occur.

Influence of the auto-transfused blood on adverse drug reaction is therefore dependent on weight of the child, plasma drug concentration at the time of cellsaver processing, and amount, speed and timing of administration of the auto-transfused blood.

The influence of cellsaver systems should be accounted for when performing pharmacological trials after cardiac surgery. Optimizing drug dosing in neonates, infants and children during and after cardiac surgery is important to improve clinical care, especially in an era where fast track recovery is becoming more important. Ideally, potential effects of the CPB- and cellsaver systems on drug concentrations should be incorporated in dosing advices [8]. The current study provides insight into the potential return of drugs through auto-transfused blood. Population pharmacokinetics can be used to determine subsequent dose adjustments of the investigated drugs $[8,10]$.

Future research should focus mainly on lipophilic anaesthetic drugs that could cause a potential adverse reaction when given to patients postoperatively through the auto-transfused blood. Also, future endeavours should aim to incorporate the results of the CPB-PHARM trial and the results of this trial into a new dosing regimen for routinely used drugs for neonates, infants and children during and after cardiac surgery. This new dosing regimen will take into account the influence of the $\mathrm{CPB}$ and the cellsaver system.

\section{Conclusion}

Depending on the drug, up to $34 \%$ of the drug concentration salvaged from the operation site is returned to the patient through autotransfusion, potentially causing unwanted drug reactions post-operatively. Additionally, influence of a cellsaver system should be considered in pharmacological research during and after congenital cardiac surgery.

\section{Abbreviations}

ASD: Atrial septal defect; CAVSD: Complete atrioventricular septal defect; Cl: Confidence interval; CPB: Cardiopulmonary bypass; ECMO: Extra corporeal membrane oxygenation; IQR: Interquartile range; IRB: Institutional review board; Kg: Kilogram; L: Litre; LC-MS/MS: Liquid chromatography-tandem mass spectrometry; LLOQ: Lower limit of quantification; Mcg: Microgram; Mg: Milligram; Min: Minute; Na: Not applicable; PICU: Paediatric intensive care unit; Rpm: Rounds per minute; TCPC: Total cavopulmonary connection; TOF: Tetralogy of fallot; ULOQ: Upper limit of quantification; Y: Years

\section{Acknowledgements}

The authors like to thank all perfusionists, paediatric cardiac anaesthesiologists and anaesthesiology assistants for collecting data and blood samples.

\section{Availability of data and materials}

The datasets used during the current study are available from the corresponding author on reasonable request.

\section{Authors' contributions}

GZ contributed to the design of the study and data collection, performed the analyses and drafted the first version of the manuscript. AvS contributed to the design and data collection and was involved in drafting the manuscript. JvR contributed to the data analyses and interpretation and critically revised the manuscript. SB contributed to the data analyses and critically revised the manuscript. AvD contributed to the data collection and interpretation and critically revised the manuscript. EW contributed to the design, data analyses and interpretation and was involved in drafting the manuscript. DT contributed to design, data analyses and interpretation and critically revised the manuscript. $A B$ contributed to design, data analyses and interpretation and critically revised the manuscript. All authors have approved the final manuscript.

\section{Ethics approval and consent to participate}

The study was approved by the Institutional research board of the Erasmus MC, registration 2011-400. Informed consent was obtained from all patients, and/or parents/legal representatives according to Dutch law.

\section{Competing interests}

The authors declare that they have no competing interest.

\section{Publisher's Note}

Springer Nature remains neutral with regard to jurisdictional claims in published maps and institutional affiliations.

\section{Author details}

${ }^{1}$ Department of Cardiothoracic Surgery, Erasmus Medical Center, Rotterdam, The Netherlands. ${ }^{2}$ Intensive Care and Department of Pediatric Surgery, Erasmus Medical Center - Sophia Children's Hospital, Rotterdam, The Netherlands. ${ }^{3}$ Department of Cardio-Thoracic Anaesthesiology, Erasmus Medical Center, Rotterdam, The Netherlands. ${ }^{4}$ Department of Biostatistics, Erasmus Medical Center, Rotterdam, The Netherlands. ${ }^{5}$ Department of Pharmacology, Erasmus Medical Center, Rotterdam, The Netherlands.

Received: 21 February 2018 Accepted: 31 May 2018

Published online: 08 June 2018

References

1. Society of Thoracic Surgeons Blood Conservation Guideline Task F, Ferraris VA, Ferraris SP, Saha SP, Hessel EA 2nd, Haan CK, Royston BD, Bridges CR, Higgins RS, Despotis G, et al. perioperative blood transfusion and blood conservation in cardiac surgery: the Society of Thoracic Surgeons and the Society of Cardiovascular Anesthesiologists clinical practice guideline. Ann Thorac Surg. 2007:83:S27-86.

2. Prieto MA, Guash S, Mendez JC, Munoz C, Planas A, Reyes G. Does use of cell saver decrease the inflammatory response in cardiac surgery? Asian Cardiovasc Thorac Ann. 2013;21:37-42. 
3. Paparella D, Whitlock R. Safety of salvaged blood and risk of coagulopathy in cardiac surgery. Semin Thromb Hemost. 2016;42:166-71.

4. Sue D, Salazar TA, Turley K, Guglielmo BJ. Effect of surgical blood loss and volume replacement on antibiotic pharmacokinetics. Ann Thorac Surg. 1989:47:857-9

5. Rohling RG, Rentsch KM, Beck-Schimmer B, Fuchs-Buder T. Risk of recurarization during retransfusion of autologous blood withdrawn after injection of muscle relaxants: a comparison of rocuronium and mivacurium. J Clin Anesth. 2003;15:85-90.

6. DrugBank [https://www.drugbank.ca/]

7. Wildschut ED, Ahsman MJ, Allegaert K, Mathot RAA, Tibboel D.

Determinants of drug absorption in different ECMO circuits. Intensive Care Med. 2010;36:2109-16.

8. van Saet A, de Wildt SN, Knibbe CA, Bogers AD, Stolker RJ, Tibboel D. The effect of adult and pediatric cardiopulmonary bypass on pharmacokinetic and pharmacodynamic parameters. Curr Clin Pharmacol. 2013:8:297-318.

9. Soulard A, Babre F, Bordes M, Meymat Y, Sztark F, Cros AM. Optimal dose of sufentanil in children for intubation after sevoflurane induction without neuromuscular block. Br J Anaesth. 2009;102:680-5.

10. Valkenburg AJ, Calvier EA, van Dijk M, Krekels EH, O'Hare BP, Casey WF, Mathot RA, Knibbe CA, Tibboel D, Breatnach CV. Pharmacodynamics and pharmacokinetics of morphine after cardiac surgery in children with and without Down syndrome. Pediatr Crit Care Med. 2016;17:930-8.

Ready to submit your research? Choose BMC and benefit from:

- fast, convenient online submission

- thorough peer review by experienced researchers in your field

- rapid publication on acceptance

- support for research data, including large and complex data types

- gold Open Access which fosters wider collaboration and increased citations

- maximum visibility for your research: over $100 \mathrm{M}$ website views per year

At BMC, research is always in progress.

Learn more biomedcentral.com/submissions 